\title{
Direct demonstration of lipid sequestration as a mechanism by which rosiglitazone prevents fatty-acid-induced insulin resistance in the rat: comparison with metformin
}

\author{
J.-M. Ye ${ }^{1}$ N. Dzamko ${ }^{1}$ M. E. Cleasby ${ }^{1}$ B. D. Hegarty ${ }^{1}$ S. M. Furler ${ }^{1}$ G. J. Cooney ${ }^{1}$ E. W. Kraegen ${ }^{1}$ \\ ${ }^{1}$ Garvan Institute of Medical Research, Darlinghurst, Sydney, Australia
}

\begin{abstract}
Aims/hypothesis. Thiazolidinediones can enhance clearance of whole-body non-esterified fatty acids and protect against the insulin resistance that develops during an acute lipid load. The present study used $\left[{ }^{3} \mathrm{H}\right]-\mathrm{R}$-bromopalmitate to compare the effects of the thiazolidinedione, rosiglitazone, and the biguanide, metformin, on insulin action and the tissue-specific fate of non-esterified fatty acids in rats during lipid infusion.

Methods. Normal rats were treated with rosiglitazone or metformin for 7 days. Triglyceride/heparin (to elevate non-esterified fatty acids) or glycerol (control) were then infused for $5 \mathrm{~h}$, with a hyperinsulinaemic clamp being performed between the 3rd and 5th hours.

Results. Rosiglitazone and metformin prevented fattyacid-induced insulin resistance (reduced clamp glucose infusion rate). Both drugs improved insulinmediated suppression of hepatic glucose output but only rosiglitazone enhanced systemic non-esterified fatty acid clearance (plateau plasma non-esterified
\end{abstract}

fatty acids reduced by $40 \%$ ). Despite this decrease in plateau plasma non-esterified fatty acids, rosiglitazone increased fatty acid uptake (two-fold) into adipose tissue and reduced fatty acid uptake into liver (by 40\%) and muscle (by 30\%), as well as reducing liver longchain fatty acyl CoA accumulation (by 30\%). Both rosiglitazone and metformin increased liver AMPactivated protein kinase activity, a possible mediator of the protective effects on insulin action, but in contrast to rosiglitazone, metformin had no significant effect on non-esterified fatty acid kinetics or relative tissue fatty acid uptake.

Conclusions/interpretation. These results directly demonstrate the "lipid steal" mechanism, by which thiazolidinediones help prevent fatty-acid-induced insulin resistance. The contrasting mechanisms of action of rosiglitazone and metformin could be beneficial when both drugs are used in combination to treat insulin resistance.

Keywords Adipose tissue - AMP-activated protein kinase $\cdot$ Biguanides $\cdot$ Fatty acid kinetics $\cdot$ Insulin resistance $\cdot$ Liver $\cdot$ Muscle $\cdot$ Thiazolidinediones
Received: 29 December 2003 / Accepted: 10 March 2004

Published online: 1 July 2004

(C) Springer-Verlag 2004

E. W. Kraegen ( $)$

Garvan Institute of Medical Research,

384 Victoria St, Darlinghurst, Sydney, NSW 2010, Australia

E-mail: e.kraegen@garvan.org.au

Tel.: +61-2-92958206, Fax: +61-2-92958201

Abbreviations: AMPK, AMP-activated protein kinase ·

${ }^{14} \mathrm{C}$-2DG, $\left[{ }^{14} \mathrm{C}\right]$-2-deoxyglucose $\cdot$ GIR, glucose infusion rate $\cdot$

HGO, hepatic glucose output - LCACoA, long-chain fatty acyl

CoA $\cdot$ PPAR, peroxisome proliferator-activated receptor .

TZDs, thiazolidinediones

\section{Introduction}

The most common form of insulin resistance in humans is associated with adiposity and abnormal lipid metabolism [1]. Thiazolidinediones (TZDs), such as rosiglitazone and pioglitazone, are effective insulinsensitising drugs now in clinical use for the treatment of Type 2 diabetes. Therapeutic effects of TZDs include the enhancement of insulin sensitivity in insulin-responsive tissues, particularly muscle and liver, and the lowering of circulating fatty acids and triglycerides [2, 3]. A contributor to the insulin-sensitising action of TZDs in muscle and liver is believed to be 
"lipid steal", which is defined as a partitioning of circulating lipids away from muscle and liver and into adipose tissue.

The "lipid steal" hypothesis is based on several lines of indirect evidence. Firstly, TZDs activate peroxisome proliferator-activated receptor (PPAR) $\gamma$, a transcription factor predominantly expressed in adipocytes. While there is some evidence that PPAR $\gamma$ plays a functional role in skeletal muscle [4], its expression levels in muscle and liver are extremely low compared with adipocytes. Secondly, TZDs lower circulating levels of lipids and lipid accumulation in non-adipose tissues including skeletal and cardiac muscle, liver and pancreatic islets $[2,5,6,7,8,9]$. However, alternative mechanisms have been proposed for the metabolic effects of TZDs, including a role for altered adipokines, particularly adiponectin [10, 11].

Although often cited, the "lipid steal" hypothesis for TZD action has to our knowledge only been directly demonstrated in a rodent model of extreme hyperinsulinaemia and obesity, the Zucker fatty rat [12]. Recently we used an acute model of insulin resistance induced by intralipid/heparin infusion [13] to show that normal rats pre-treated with pioglitazone were protected against the development of insulin resistance in muscle and liver. Although these findings further implicated anti-hyperlipidaemic effects of TZDs in their insulin-sensitising action, the study did not directly demonstrate the "lipid steal" mechanism. Recently, a $\left[{ }^{3} \mathrm{H}\right]-\mathrm{R}$-bromopalmitate tracer technology has been developed, which allows the in vivo kinetics (including measurement of clearance and distribution) of fatty acids into specific tissues to be determined [14].

A primary aim of the present study was to investigate the effect of rosiglitazone on tissue-specific fatty acid uptake and distribution during triglyceride/heparin infusion and clamp. The second aim was to examine the specificity of the "lipid steal" mechanism by comparing the effect of rosiglitazone with that of metformin. Metformin is a biguanide anti-hyperglycaemic drug known to reduce the incidence of diabetes in humans with obesity [15]. Its mechanism of action is unclear, but interestingly it was recently shown to enhance activity of AMP-activated protein kinase (AMPK) in isolated muscle and liver cells in vitro [16]. The AMPK pathway and its interactions were recently reviewed $[17,18]$, but it is noteworthy that prior activation of AMPK has been associated with insulin sensitisation in muscle and/or liver [19, 20]. However, we are not aware of any studies examining whether or not metformin's anti-hyperglycaemic actions are associated with altered in vivo fatty acid tissue distribution.

\section{Materials and methods}

General design. Normal rats pre-treated with rosiglitazone and metformin for 7 days were infused with lipid to examine the protective effects of these agents against acute fatty-acid- induced insulin resistance, with specific analysis of their effects on fatty acid kinetics. Insulin sensitivity was assessed by euglycaemic-hyperinsulinaemic clamp incorporating a radiolabelled glucose tracer with simultaneous measurement of lipid clearance using a radiolabelled fatty acid tracer. All experimental procedures were approved by the Animal Experimentation Ethics Committee (Garvan Institute/St Vincent's Hospital) and were in accordance with the National Health and Medical Research Council of Australia Guidelines on Animal Experimentation.

Animal experiments. Male Wistar rats, aged 10 to 12 weeks (supplier: Animal Resources Centre, Perth, WA, Australia), were maintained at $22 \pm 0.5{ }^{\circ} \mathrm{C}$ with a 12 by 12 -h day/night cycle for one week. They had free access to food (standard chow diet). After 1 week of acclimatisation, the animals were given rosiglitazone $\left(4 \mathrm{mg} \cdot \mathrm{kg}^{-1} \cdot \mathrm{day}^{-1}\right)$, metformin $\left(120 \mathrm{mg} \cdot \mathrm{kg}^{-1}\right.$ $\mathrm{day}^{-1}$ ) or vehicle (control). This was done by gavage once daily between 15.00 and 17.00 hours for 1 further week. Six to seven days prior to the acute study, the left carotid artery and right jugular vein of some rats were cannulated under halothane anaesthesia. Rats were handled daily to minimise stress and only those rats with fully recovered body weight were used for the final study.

On the day of the acute study, rats were fasted for 5 to $7 \mathrm{~h}$ and received a final dose of drug at approximately 09.00 hours. Rats were then studied under one of three study conditions: (i) basal, (ii) $3.75 \mathrm{~h}$ of lipid infusion alone, and (iii) $5 \mathrm{~h}$ of lipid infusion including a hyperinsulinaemic-euglycaemic clamp in the last $2 \mathrm{~h}$. For basal studies, rats were killed by pentobarbitone injection between 12.00 and 13.00 hours. Liver, muscle and subcutaneous fat were immediately taken and quickly freeze-clamped. For study conditions (ii) and (iii) above, rats were acclimatised for 30 to 40 min following connection of the cannulae to sampling and infusion syringes. For study condition (ii), triglyceride emulsion (Intralipid, Baxter Healthcare, Sydney, NSW, Australia, $0.5 \mathrm{ml} / \mathrm{h}$ ) plus heparin $(40 \mathrm{U} / \mathrm{h})$ was infused, with a bolus of $\left[{ }^{14} \mathrm{C}\right]-2$-deoxyglucose $\left({ }^{14} \mathrm{C}-2 \mathrm{DG}\right) \mathrm{ad}-$ ministered via the jugular line after $3 \mathrm{~h}$ of infusion, followed by $45 \mathrm{~min}$ of frequent blood sampling [21] to determine glucose turnover. Tissues were collected subsequently as described above. For study condition (iii), rats underwent $5 \mathrm{~h}$ of triglyceride/heparin infusion with a euglycaemic-hyperinsulinaemic clamp (450-500 mU/l) between the 3rd and 5th hours. At the 75 th min of the clamp, a bolus of ${ }^{14} \mathrm{C}-2 \mathrm{DG}$ was injected to estimate whole-body glucose disposal, hepatic glucose output (HGO) and tissue glucose uptake [22]. Sixteen min prior to the end of the clamp, $\left[{ }^{3} \mathrm{H}\right]-\mathrm{R}$-bromopalmitate tracer was administered via the carotid line as previously described [14] to measure fatty acid clearance rate and uptake in individual tissues. Rats infused with glycerol $(0.4 \mathrm{ml} / \mathrm{h})$ and heparin $(40 \mathrm{U} / \mathrm{h})$ were used as controls for each of the above studies replacing the triglyceride and heparin infusion.

Determination of tissue tracer content. With regard to fatty acid tracer, tissue samples were homogenised in chloroform:methanol (2:1) using a glass homogeniser. An aliquot of this homogenate was taken to determine the total ${ }^{3} \mathrm{H}$ activity. Tissue fatty acid uptake was estimated from plasma tracer disappearance and tissue ${ }^{3} \mathrm{H}$ activity as previously described [14]. Details of the method for extraction of the glucose tracer and related calculations have been published previously [23].

Metabolites and hormone measurements. Plasma glucose was determined using a glucose analyser (YSI 2300, YSI, Yellow Springs, Ohio, USA). Plasma NEFAs were determined spectrophotometrically using an acyl-CoA oxidase-based colorimetric kit (NEFA-C; WAKO Pure Chemical Industries, Osaka, 
Japan). Plasma triglyceride concentrations were measured using enzymatic colorimetric methods (Triglyceride INT, procedure 336 and GPO Trinder, Sigma-Aldrich, St Louis, Mo., USA). Plasma leptin, adiponectin and insulin were determined by radioimmunoassay using commercial kits for the rat (Linco Research, St Charles, Mo., USA). Tissue triglycerides were extracted [24] and measured by a Peridochrom Triglyceride GPO-PAP kit (Roche Diagnostics, Indianapolis, Ind., USA). Total tissue long-chain acyl CoA (LCACoA) concentrations were determined by a fluorescence spectrophotometric assay based on a method that has been previously described [25].

Assays of AMP-activated protein kinase activity. The activity of AMPK in tissue extracts was assayed in a buffer containing $40 \mathrm{mmol} / \mathrm{l}$ HEPES buffer (pH 7.0), $200 \mu \mathrm{mol} / \mathrm{l}$ AMP, $200 \mu \mathrm{mol} / \mathrm{l}$ ATP, $80 \mathrm{mmol} / \mathrm{l} \mathrm{NaCl}, 8 \%$ glycerol, $0.8 \mathrm{mmol} / \mathrm{l}$ EDTA, $5 \mathrm{mmol} / \mathrm{l} \mathrm{MgCl}, 0.8 \mathrm{mmol} / \mathrm{l}$ dithiothreitol and $200 \mu \mathrm{mol} / 1\left[\gamma^{32}{ }^{32}\right.$ ]ATP [26]. This was done for $6 \mathrm{~min}$ at $37{ }^{\circ} \mathrm{C}$. As a substrate we used the AMPK-specific AMARA peptide [26] (Auspep, Victoria, Australia). Some AMPK data from the metformin-lipid group have also been incorporated in a more specific study of metformin action but are included here to facilitate comparison with rosiglitazone.

Statistical analysis. All results are presented as means \pm SE. A repeated measure analysis of the variance (repeated ANOVA) was used to assess results measured at consecutive multiple time points. Here a two-way design was used to incorporate additional effects of different experimental groups followed by a post-hoc (PLSD) test to compare two individual groups. Other comparisons were made using a one-way ANOVA followed by a PLSD test to compare two individual groups. The Macintosh Statview program (Abacus Concepts-Brain Power, Cary, N.C., USA) was used to perform the statistics. Group sizes ranged from five to seven rats unless otherwise specified. A $p$ value of 0.05 was considered significant.

\section{Results}

Effects on metabolic parameters in the basal state and during lipid infusion. Body weight and basal plasma glucose of normal rats were not significantly changed by one week's treatment with rosiglitazone or metformin (final body weights: control $378 \pm 5$, rosiglitazone $366 \pm 4$, metformin $362 \pm 5 \mathrm{~g}$; plasma glucose: control $8.1 \pm 0.1$, rosiglitazone $7.7 \pm 0.2$, metfor$\min 7.7 \pm 0.1 \mathrm{mmol} / \mathrm{l})$. Other basal plasma parameters are shown in Figure 1. Significant changes in the rosiglitazone-treated group were a two-fold rise in plasma concentrations of adiponectin and a $30 \%$ decrease in plasma triglycerides. Insulin and NEFA were similar to the control group. Compared with the control group, rats treated with metformin had significantly $(\sim 30 \%)$ lower plasma insulin levels but similar plasma levels of adiponectin, triglycerides and NEFA. Epididymal fat pad masses were measured as an indication of the degree of adiposity between groups; values were not significantly different, although there was a tendency towards lower depot size in the metformin group (control 1.3 \pm 0.1 ; rosiglitazone 1.3 \pm 0.1 ; metformin $1.1 \pm 0.1 \mathrm{~g}$ ).

Figure 2 shows the effect of rosiglitazone and metformin on plasma levels of NEFA andtriglyce-
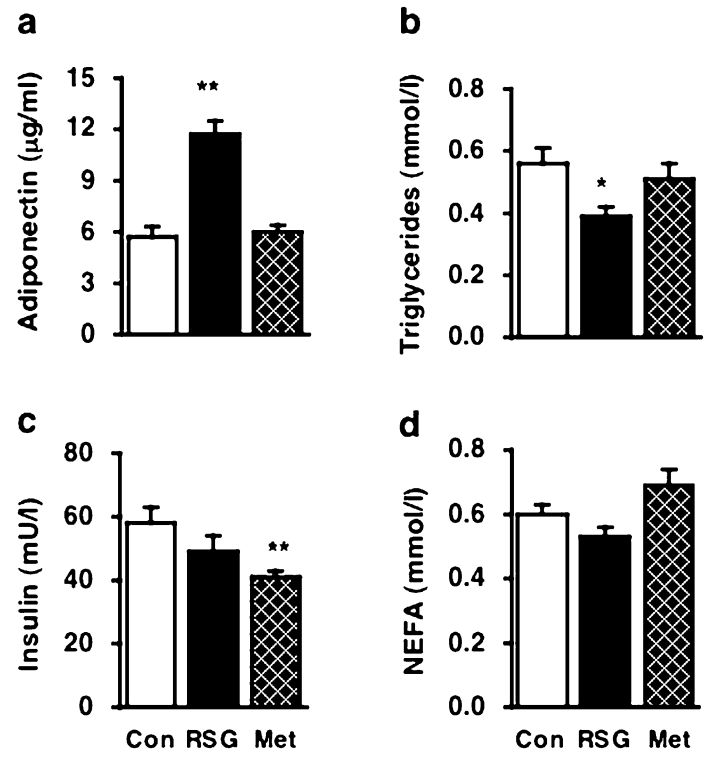

Fig. 1. Effects of 7 days of treatment with rosiglitazone (RSG) or metformin (Met) on basal plasma levels of adiponectin (a), triglycerides (b), insulin (c) and NEFA (d) in normal rats. Data are presented as means \pm SE. $n \geq 7$ per group; $* p<0.05$, $* * p<0.01$ vs controls
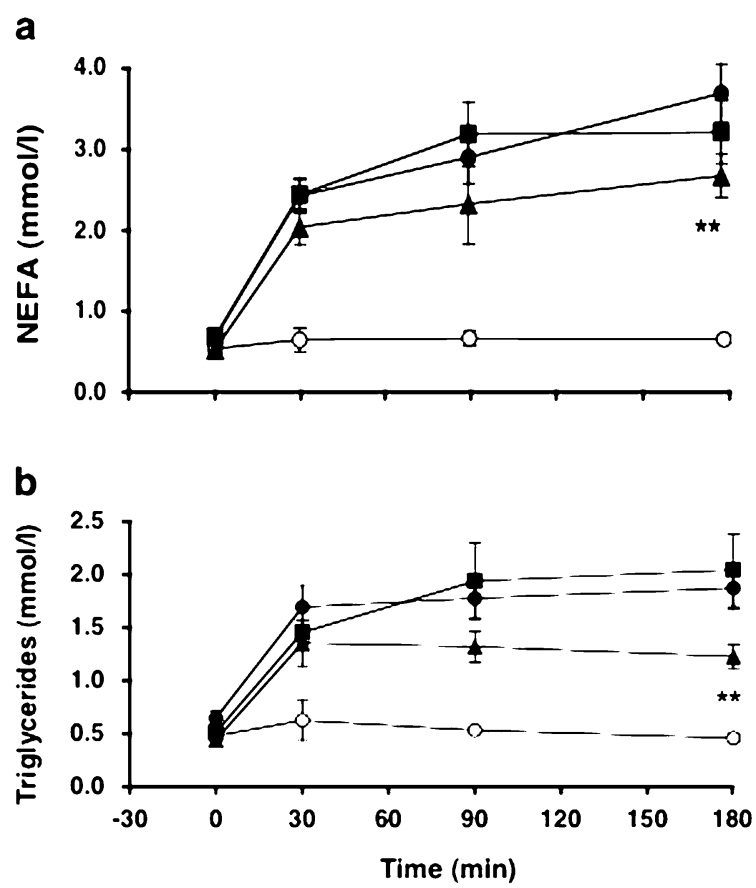

Fig. 2. Effects of 7 days of treatment with rosiglitazone or metformin on plasma levels of NEFA (a) and triglycerides (b) during acute (3-h) triglyceride/heparin infusion in normal rats. Open circles: control-glycerol; closed circles: control-lipid; closed triangles: rosiglitazone-lipid; closed squares: metformin-lipid. In all lipid infusion groups, plasma triglyceride and fatty acid levels were significantly higher than in the control-glycerol group $(p<0.05)$. $* * p<0.01$ rosiglitazone-lipid vs control-lipid 


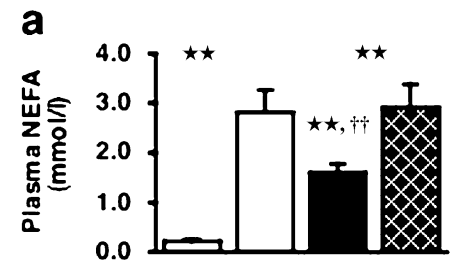

b
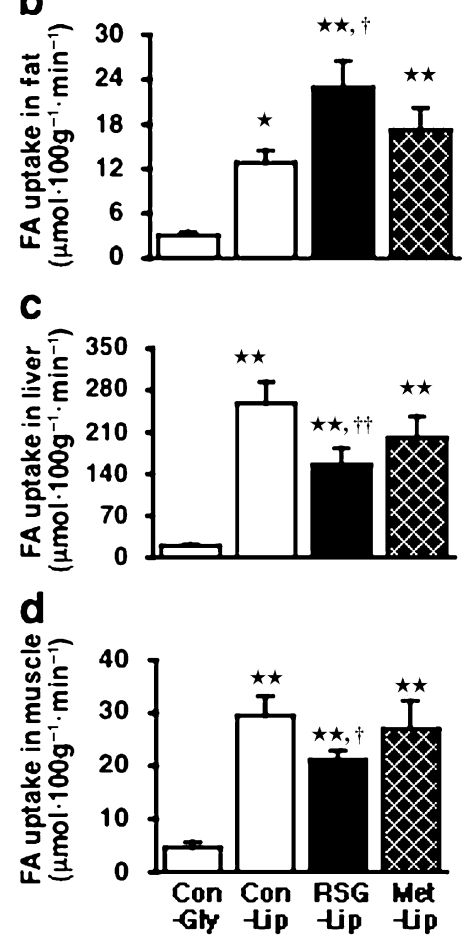

Fig. 3. Effects of 7 days of treatment with rosiglitazone or metformin on plasma NEFA levels (a) and fatty acid (FA) uptake in fat (b), liver (c) and muscle (d) during acute triglyceride/heparin infusion and hyperinsulinaemic-euglycaemic clamp in normal rats. Con-Gly, control-glycerol; Con-Lip, control-lipid; RSG-Lip, rosiglitazone-lipid; Met-Lip, metformin-lipid. *, $p<0.05$, **, $p<0.01$ vs control-glycerol; $\dagger, p<0.05, \dagger \dagger, p<0.01$ vs control-lipid

ride during the 3-h lipid infusion and prior to the hyperinsulinaemic-euglycaemic clamp. Compared with the glycerol-infused control group (control-glycerol group), plasma levels of triglyceride and NEFA were elevated in the control-lipid group by 4 - and 5.5-fold respectively $(p<0.01)$. However, compared with the control-lipid group, plasma triglyceride and NEFA levels were reduced at $3 \mathrm{~h}$ in the rosiglitazone-lipid group by 30 to $35 \%$ ( $p<0.05$ vs control-lipid). In contrast, plasma triglyceride and NEFA levels in the metformin-lipid group were not significantly different from the control-lipid group. There were no significant differences in plasma insulin levels between all four groups after $3 \mathrm{~h}$ of lipid or glycerol infusion (data not shown).

Effects on fatty acid kinetics. To assess the effects of rosiglitazone and metformin on fatty acid kinetics in

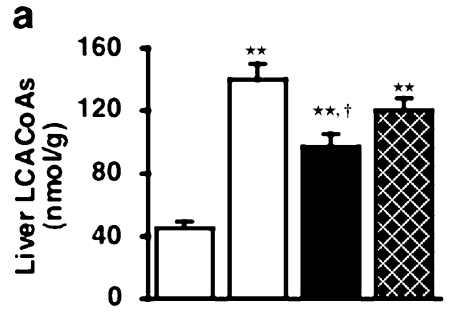

b

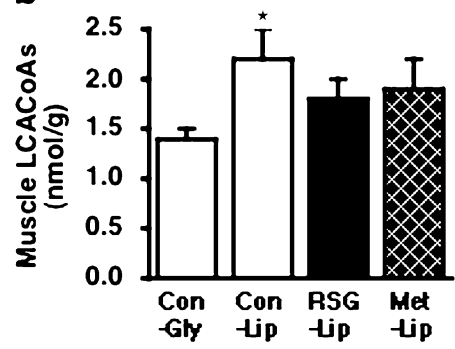

Fig. 4. Effects of 7 days of treatment with rosiglitazone or metformin on liver (a) and muscle (b) long-chain acyl CoA (LCACoA) content after acute triglyceride/heparin infusion and hyperinsulinaemic-euglycaemic clamp in normal rats. Con-Gly, control-glycerol; Con-Lip, control-lipid; RSG-Lip, rosiglitazone-lipid; Met-Lip, metformin-lipid. *, $p<0.05$, $* *, p<0.01$ vs control-glycerol; $\dagger, p<0.05$ vs control-lipid

relation to insulin sensitivity, the lipid and glycerol infusions were extended to $5 \mathrm{~h}$ with a hyperinsulinaemic-euglycaemic clamp being performed in the last $2 \mathrm{~h}$. The relative pattern of plasma NEFA levels during the steady state of the hyperinsulinaemic clamp was similar to that with lipid infusion alone. Compared with the control-lipid group, plasma NEFA levels were decreased by $44 \%$ in the rosiglitazone-lipid group $(p<0.05$ vs control-lipid) (Fig. 3$)$. In contrast to rosiglitazone, metformin did not have a significant effect on plasma NEFA levels during the hyperinsulinaemic clamp. Adipose tissue, liver and muscle fatty acid uptake were substantially increased in all lipid infusion groups compared with the control-glycerol group. However, compared with the control-lipid group, fatty acid uptake in adipose tissue in the rosiglitazone-lipid group was enhanced by about 1.8 -fold, accompanied by decreased fatty acid uptake in both liver $(\sim 40 \%)$ and muscle $(\sim 30 \%)$. There were no significant differences in fatty acid uptake by adipose tissue, muscle or liver between the metformin- lipid and control-lipid groups.

Figure 4 shows the content of LCACoAs in liver and muscle. Compared with the control-glycerol group, LCACoAs were increased three-fold in the control-lipid group in liver and 1.6-fold in muscle. Consistent with a reduced fatty acid uptake in liver, LCACoA content was about $30 \%$ lower in the rosiglitazone-lipid group than in the control-lipid group, whereas the reduction in LCACoAs in metforminlipid was not statistically significant. LCACoAs in muscle in the rosiglitazone-lipid and metformin-lipid groups were not significantly different from those 

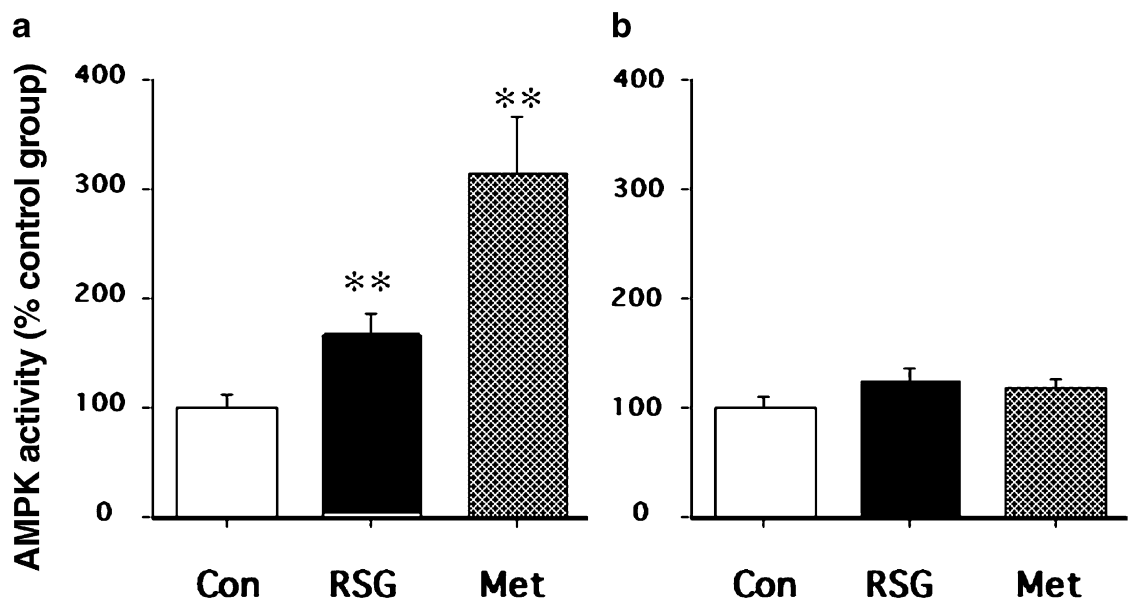

Fig. 5. Effects of 7 days of treatment with rosiglitazone or metformin on liver (a) and muscle (b) AMPK activity. $* * p<0.01$ vs control group. Con, control; RSG, rosiglitazone; Met, metformin

Table 1. Metabolic changes during 5-h intralipid/heparin infusion plus final 2-h hyperinsulinaemic-euglycaemic clamp

\begin{tabular}{lcccc}
\hline & Control-glycerol & Control-lipid & Rosiglitazone-lipid & Metformin-lipid \\
\hline Plasma glucose $(\mathrm{mmol} / \mathrm{l})$ & $8.0 \pm 0.3$ & $8.3 \pm 0.1$ & $8.0 \pm 0.2$ & $8.4 \pm 0.2$ \\
Plasma insulin $(\mathrm{mU} / \mathrm{l})$ & $488 \pm 45$ & $514 \pm 65$ & $456 \pm 90$ & $499 \pm 50$ \\
GIR $\left(\mathrm{mg} \cdot \mathrm{kg}^{-1} \cdot \mathrm{min}^{-1}\right)$ & $29.9 \pm 0.7$ & $20.9 \pm 0.7 \mathrm{a}$ & $29.5 \pm 2.0^{\mathrm{c}}$ & $30.1 \pm 2.5^{\mathrm{c}}$ \\
Rd $\left(\mathrm{mg} \cdot \mathrm{kg}^{-1} \cdot \mathrm{min}^{-1}\right)$ & $28.6 \pm 0.9$ & $25.7 \pm 1.8$ & $30.8 \pm 1.1$ & $27.9 \pm 3.3$ \\
Muscle Rg' $\left(\mu \mathrm{mol}^{100} \mathrm{~g}^{-1} \cdot \mathrm{min}^{-1}\right)$ & $39.2 \pm 5.1$ & $30.8 \pm 3.3$ & $37.2 \pm 3.5$ & $24.6 \pm 5.0^{\mathrm{d}}$ \\
HGO $\left(\mathrm{mg} \cdot \mathrm{kg}^{-1} \cdot \mathrm{min}^{-1}\right)$ & $-1.26 \pm 0.9$ & $6.2 \pm 1.6^{\mathrm{a}}$ & $1.2 \pm 2.1^{\mathrm{b}}$ & $-2.1 \pm 1.0^{\mathrm{c}}$ \\
\hline
\end{tabular}

Statistical comparisons are: ${ }^{\mathrm{a}}, p<0.01$ vs control-glycerol; ${ }^{\mathrm{b}}, p<0.05$, c,$p<0.01$ vs control-lipid; ${ }^{\mathrm{d}} p<0.05$ vs rosiglitazone-lipid. GIR, glucose infusion rate; HGO, hepatic glucose output; Rd, rate of glucose disposal; Rg', tissue glucose metabolic index

in the control-lipid group ( $p>0.05$ vs control-lipid). Final liver and muscle triglyceride levels between groups showed a similar pattern to the LCACoA data, with liver triglyceride $24 \%$ lower in the rosiglitazonelipid group than in the control-lipid group $(p<0.05)$, whereas metformin did not affect liver triglycerides (control-glycerol 22 \pm 2 , control-lipid 33 \pm 4 , rosiglitazone-lipid $25 \pm 3$, metformin-lipid $34 \pm 3 \mathrm{~g}$ ). Muscle triglycerides were approximately 50\% higher $(p<0.05)$ in the three lipid-infused groups than in the control-glycerol group, but no effect of drug treatment was observed (data not shown).

Effects on insulin sensitivity. As shown in Table 1, plasma levels of glucose and insulin were well matched among all groups during the hyperinsulinaemic clamp. Whole-body insulin sensitivity was indicated by the steady-state glucose infusion rate (GIR) required for maintaining euglycaemia. Compared with the controlglycerol group, lipid infusion (control-lipid) significantly suppressed GIR (by $30 \%$ ), indicating the development of insulin resistance. This was not observed in the rosiglitazone-lipid group, in which GIR remained at a similar level to the control-glycerol group. Despite its lack of effect in reducing plasma NEFA levels and liver LCACoA content, pre-treatment with metformin preserved GIR to a similar extent to rosiglitazone, indicating that it was as effective as rosiglitazone in preventing the development of lipid-induced insulin resistance. Although the rate of glucose disposal did not differ significantly among the groups, glucose uptake in muscle tended to be lower in the control-lipid group, a trend which was opposed in the rosiglitazone-lipid but not in the metformin-lipid group.

During the lipid or glycerol infusion alone, HGO was similar in all four groups, being 11.5 \pm 1.0 , $13.8 \pm 1.5,12.3 \pm 0.5$ and $13.9 \pm 1.2 \mathrm{mg} \cdot \mathrm{kg}^{-1} \cdot \mathrm{min}^{-1}$ for control-glycerol, control-lipid, rosiglitazone-lipid and metformin-lipid respectively $(p>0.05, n=6$ per group). During lipid or glycerol infusion plus the hyperinsulinaemic clamp, HGO was completely suppressed in the control-glycerol group, whereas in the control-lipid group the ability of insulin to suppress HGO was significantly reduced (Table 1 ), indicating insulin resistance in the liver. In the rosiglitazonelipid and metformin-lipid groups, the ability of insulin to suppress HGO was significantly improved.

Effects on AMP activated protein kinase activity. To examine a possible mechanism for the effect of rosiglitazone and metformin on liver, we further examined the activity of AMPK in rats treated with or without rosiglitazone and metformin. Although neither rosiglitazone nor metformin had any significant effects on AMPK activity in muscle (Fig. 5), the activity of AMPK in liver was increased (1.7-fold, 
$p<0.01$ vs control) in rosiglitazone-treated rats. The effect of metformin was even greater (3-fold increase, $p<0.01$ vs control).

\section{Discussion}

The present study directly demonstrates that the ability of rosiglitazone, a TZD, to protect against insulin resistance caused by an acute elevation of fatty acids is associated with altered fatty acid distribution and uptake in muscle, liver and adipose tissue. The results confirm recent studies $[13,27]$ that in normal rats pretreated with a TZD, the ability to clear systemic fatty acids is substantially augmented (by $\sim 62 \%$ ) in the face of an elevation in fatty acids produced by triglyceride/heparin infusion. The current study goes on to show that enhancement of whole-body fatty acid uptake by TZD pre-treatment is associated with a substantial increase ( 2-fold) in the flux of circulating fatty acids into adipose tissue, with decreased uptake into liver and muscle. In parallel with reduced fatty acid fluxes into liver and muscle, insulin action in these tissues did not deteriorate during the lipid infusion in rosiglitazone-treated rats. Altered lipid kinetics were not a pre-requisite for insulin sensitisation, however, as evidenced by the contrasting effects of rosiglitazone and metformin in our study. Although metformin pre-treatment also preserved insulin sensitivity during lipid infusion, this had no effect on NEFA clearance and tissue distribution, suggesting that effects on fatty acid uptake are specific to TZDs. Thus there are distinct differences in the metabolic consequences of rosiglitazone and metformin treatment. However, both compounds increased AMPK activity in liver, implying that activation of this enzyme may mediate some of the insulin-sensitising effects of both drugs in liver.

Our earlier work using the high-fat fed rat model of insulin resistance showed that the insulin-sensitising action of TZDs is accompanied by attenuated lipid accumulation in muscle and liver [5, 28]. It has been proposed that TZDs act primarily in adipocytes and sequester circulating lipids in adipose tissue [2]. This would reduce lipid supply to muscle and liver, thus improving insulin action in these tissues [29]. With the use of the bromopalmitate fatty acid tracer technique, the present work clearly shows an enhancement of NEFA clearance from the circulation following treatment with rosiglitazone, consistent with an earlier study [13]. More importantly, the substantial increase that we have demonstrated in fatty acid uptake in adipose tissue strongly suggests that this tissue is a major recipient of the increased NEFA disposal seen in rosiglitazone-treated rats. Consistent with the reduction in fatty acid flux into liver, the increment in hepatic LCACoA (an intracellular form of fatty acids) and triglycerides was reduced, and the ability of insu- lin to suppress $\mathrm{HGO}$ was maintained. A similar relationship between fatty acid uptake and insulin sensitivity was also identified in muscle, although there was no significant difference in LCACoA or triglyceride in this tissue. Thus, the present study directly demonstrates that the "lipid steal" mechanism helps prevent insulin resistance from developing in muscle and liver in the presence of an acute rise in circulating NEFA concentrations.

Although a similar mechanism has been observed in obese Zucker rats treated with a TZD [12], the implications of the "lipid steal" mechanism in normal rats are different from those in obese Zucker rats. In the present study, the animals were normal in insulin sensitivity and lipid metabolism. Under these conditions, the increases in fatty acid clearance and disposal in adipose tissue during acute lipid loading indicate that TZDs are able to protect insulin action in muscle and liver by reducing lipid oversupply to these tissues and that they do this even in the normal physiological state. This could be viewed as an enhancement of the "buffering" ability of adipose tissue in normal systemic lipid regulation [30], presumably by enhanced expression of proteins determining adipose tissue lipogenesis, which is an established effect of PPAR $\gamma$ activation [31].

While our studies reveal a substantial increase in fatty acid clearance into adipose tissue, there is no evidence that fatty acid clearance into muscle or liver is reduced to a significant extent by prior rosiglitazone treatment. This is evidenced by the fact that the reduction in fatty acid uptake into muscle and liver (30-40\%) was in proportion with the reduction in plateau fatty acid levels during lipid infusion as achieved in rosiglitazone-treated rats compared with untreated rats. This suggests that altered systemic fatty acid kinetics in rosiglitazone-treated rats are largely determined by adipose tissue rather than muscle and liver. The question of whether TZDs exert direct effects in muscle and liver has been controversial. In the case of muscle, it has been elegantly addressed by two recent studies of metabolic responses in muscle-specific PPAR $\gamma$ knock-out mouse models [4, 32]. There are significant differences between these two models. Interestingly, however, one group found that the lack of functioning PPAR $\gamma$ in muscle did not alter the wholebody or muscle insulin-sensitising actions of rosiglitazone. This supports the idea that TZD effects in muscle are the result of indirect action, as could be provided by the "lipid steal" mechanism revealed here. Nevertheless, the possibility that PPAR $\gamma$ activation by rosiglitazone in muscle has some influence on local and systemic fatty acid metabolism cannot be ruled out, particularly as the knock-out models have some disturbances of lipid metabolism, e.g. increased adiposity [32].

Our study also revealed a lower plasma triglyceride plateau in the rosiglitazone-treated group than in the 
untreated and metformin-treated group. The intralip$\mathrm{id} /$ heparin technique is commonly used to study fatty acid clearance, but in vivo study of the fate of triglycerides derived from lipoprotein particles requires a different technique now being developed [33]. Since rosiglitazone can increase LPL expression [34], it is possible that rosiglitazone increased hydrolysis of triglyceride, but the lower plasma fatty acid levels argue against this. Another possibility is that TZDs inhibited Apo C-III expression [35], which could perhaps lead to enhanced removal of triglyceride-rich particles without lipolysis [36]. These speculative possibilities do not invalidate the methodology used here for studying tissue-specific NEFA clearance. However, it is conceivable that within-tissue modulation of NEFA uptake by competing non-lipolytic processes occurs, although we are not aware of any evidence for this.

One similarity in the effects of rosiglitazone and metformin was their influence on AMPK activity in liver. Given the possibility that AMPK activation may play a role in increasing insulin action $[19,20]$, this provides a possible common link in the protective effects of the two agents in preventing insulin resistance from developing in the liver. A possible mediator of enhanced AMPK activity induced by rosiglitazone is increased plasma adiponectin [10], a potential candidate mechanism for the insulin-sensitising action of TZDs which might act in addition to "lipid steal". In support of this, we previously found that the protective effect of pioglitazone on insulin sensitivity in the liver during triglyceride/heparin infusion was strongly correlated with elevated concentrations of plasma adiponectin [13]. In high-fat fed rats treated with different PPAR $\gamma$ agonists including rosiglitazone, improved muscle and liver insulin sensitivity was also accompanied by dramatic increases in plasma concentrations of adiponectin [37]. Consistently, the present study showed a two-fold increase in plasma concentrations of adiponectin after rosiglitazone pre-treatment. As AMPK is thought to be a key target in the signal transduction pathways activated by adiponectin in muscle and liver [11], this could explain the AMPK activation observed following treatment with rosiglitazone. Metformin was even more potent than rosiglitazone in its activation of liver AMPK activity. In contrast with rosiglitazone, however, this change was not associated with elevated adiponectin. It is likely that the AMPK-activating effects of metformin are mediated by direct intracellular mechanisms, since metformin activated AMPK in isolated hepatocytes [16]. Further work will be necessary to establish whether AMPK activation is indeed a factor contributing to the preservation of insulin-induced suppression of $\mathrm{HGO}$ by rosiglitazone and metformin.

In summary, both rosiglitazone and metformin oppose insulin resistance induced by an acute lipid load. The insulin-sensitising effect of rosiglitazone is accompanied by a partitioning of fatty acid uptake into adipose tissue and away from the liver and muscle (directly demonstrating the "lipid steal" hypothesis). These changes in lipid metabolism clearly contrast with the lack of similar effects produced by metformin. These complementary actions of rosiglitazone and metformin suggest a possible use in combination for the treatment of lipid-associated insulin resistance. The present study also reveals that activation of AMPK is a common factor in the effects of rosiglitazone and metformin in the liver. Whether this is a key factor in the beneficial metabolic effects of these drugs in the liver will need to be determined by further study.

Acknowledgements. This study was supported by grants from GlaxoSmithKline, the National Health \& Medical Research Council of Australia and the Juvenile Diabetes Research Foundation. The authors wish to thank Drs Steve Smith and Nick Oakes for expert comments. AstraZeneca, Mölndal, Sweden supplied ${ }^{3} \mathrm{H}$-bromopalmitate tracer for this study.

\section{References}

1. McGarry JD (1992) What if Minkowski had been ageusic? An alternative angle on diabetes. Science 258:766-770

2. Spiegelman BM (1998) PPAR-gamma: adipogenic regulator and thiazolidinedione receptor. Diabetes 47:507-514

3. Moller DE (2001) New drug targets for type 2 diabetes and the metabolic syndrome. Nature 414:821-827

4. Hevener AL, He W, Barak Y et al. (2003) Muscle-specific PPARg deletion causes insulin resistance. Nat Med 9:14911497

5. Oakes ND, Kennedy CJ, Jenkins AB, Laybutt DR, Chisholm DJ, Kraegen EW (1994) A new antidiabetic agent, BRL 49653, reduces lipid availability and improves insulin action and glucoregulation in the rat. Diabetes 43:1203-1210

6. Oakes ND, Bell KS, Furler SM et al. (1997) Diet-induced muscle insulin resistance in rats is ameliorated by acute dietary lipid withdrawal or a single bout of exerciseparallel relationship between insulin stimulation of glucose uptake and suppression of long-chain fatty acyl-CoA. Diabetes 46:2022-2028

7. Zhou YT, Grayburn P, Karim A et al. (2000) Lipotoxic heart disease in obese rats: implications for human obesity. Proc Natl Acad Sci USA 97:1784-1789

8. Shimabukuro M, Zhou YT, Lee Y, Unger RH (1998) Troglitazone lowers islet fat and restores beta cell function of Zucker diabetic fatty rats. J Biol Chem 273:3547-3550

9. Higa M, Zhou YT, Ravazzola M, Baetens D, Orci L, Unger RH (1999) Troglitazone prevents mitochondrial alterations, beta cell destruction, and diabetes in obese prediabetic rats. Proc Natl Acad Sci USA 96:11513-11518

10. Combs TP, Wagner JA, Berger J et al. (2002) Induction of adipocyte complement-related protein of 30 kilodaltons by PPARgamma agonists: a potential mechanism of insulin sensitization. Endocrinology 143:998-1007

11. Yamauchi T, Kamon J, Ito $Y$ et al. (2003) Cloning of adiponectin receptors that mediate antidiabetic metabolic effects. Nature 423:762-769

12. Oakes ND, Thalen PG, Jacinto SM, Ljung B (2001) Thiazolidinediones increase plasma-adipose tissue FFA exchange capacity and enhance insulin-mediated control of systemic FFA availability. Diabetes 50:1158-1165 
13. Ye JM, Frangioudakis G, Iglesias MA et al. (2002) Prior thiazolidinedione treatment preserves insulin sensitivity in normal rats during acute fatty acid elevation: role of the liver. Endocrinology 143:4527-4535

14. Oakes ND, Kjellstedt A, Forsberg GB et al. (1999) Development and initial evaluation of a novel method for assessing tissue-specific plasma free fatty acid utilization in vivo using (R)-2-bromopalmitate tracer. J Lipid Res 40:11551169

15. Knowler WC, Barrett-Connor E, Fowler SE et al. (2002) Reduction in the incidence of type 2 diabetes with lifestyle intervention or metformin. N Engl J Med 346:393-403

16. Zhou G, Myers R, Li Y et al. (2001) Role of AMP-activated protein kinase in mechanism of metformin action. J Clin Invest 108:1167-1174

17. Ruderman NB, Saha AK, Kraegen EW (2003) Minireview: malonyl CoA, AMP-activated protein kinase, and adiposity. Endocrinology 144:5166-5171

18. Kemp BE, Stapleton D, Campbell DJ et al. (2003) AMP-activated protein kinase, super metabolic regulator. Biochem Soc Trans 31:162-168

19. Fisher JS, Gao J, Han DH, Holloszy JO, Nolte LA (2002) Activation of AMP kinase enhances sensitivity of muscle glucose transport to insulin. Am J Physiol 282:E18-E23

20. Iglesias MA, Ye JM, Frangioudakis G et al. (2002) AICAR administration causes an apparent enhancement of muscle and liver insulin action in insulin resistant high-fat fed rats. Diabetes 51:2886-2894

21. Kraegen EW, James DE, Jenkins AB, Chisholm DJ (1985) Dose-response curves for in vivo insulin sensitivity in individual tissues in rats. Am J Physiol 248:E353-E362

22. Kraegen EW, James DE, Storlien LH, Burleigh KM, Chisholm DJ (1986) In vivo insulin resistance in individual peripheral tissues of the high fat fed rat: assessment by euglycaemic clamp plus deoxyglucose administration. Diabetologia 29:192-198

23. James DE, Burleigh KM, Kraegen EW (1985) Time dependence of insulin action in muscle and adipose tissue in the rat in vivo. An increasing response in adipose tissue with time. Diabetes 34:1049-1054

24. Bligh EG, Dyer WJ (1959) A rapid method of total lipid extraction and purification. Can J Biochem Physiol 37:911-917

25. Antinozzi PA, Segall L, Prentki M, McGarry JD, Newgard CB (1998) Molecular or pharmacologic perturbation of the link between glucose and lipid metabolism is without effect on glucose-stimulated insulin secretion. A re-evaluation of the long-chain acyl-CoA hypothesis. J Biol Chem 273: 16146-16154
26. Davies SP, Carling D, Hardie DG (1989) Tissue distribution of the AMP-activated protein kinase, and lack of activation by cyclic-AMP-dependent protein kinase, studied using a specific and sensitive peptide assay. Eur J Biochem 186:123-128

27. Hevener AL, Reichart D, Janez A, Olefsky J (2001) Thiazolidinedione treatment prevents free fatty acid-induced insulin resistance in male Wistar rats. Diabetes 50:23162322

28. Ye JM, Doyle PJ, Iglesias MA, Watson DG, Cooney GJ, Kraegen EW (2001) Peroxisome proliferator-activated receptor (PPAR)-alpha activation lowers muscle lipids and improves insulin sensitivity in high fat-fed rats: comparison with PPAR-gamma activation. Diabetes 50:411-417

29. Kraegen E, Cooney G, Ye JM, Furler S (2002) Peroxisome proliferator activated receptors, fatty acids and muscle insulin resistance. J R Soc Med 95:14-22

30. Frayn KN (2002) Adipose tissue as a buffer for daily lipid flux. Diabetologia 45:1201-1210

31. Way JM, Harrington WW, Brown KK et al. (2001) Comprehensive messenger ribonucleic acid profiling reveals that peroxisome proliferator-activated receptor gamma activation has coordinate effects on gene expression in multiple insulin-sensitive tissues. Endocrinology 142:12691277

32. Norris AW, Chen L, Fisher SJ et al. (2003) Muscle-specific PPARgamma-deficient mice develop increased adiposity and insulin resistance but respond to thiazolidinediones. J Clin Invest 112:608-618

33. Thalen P, Frangioudakis G, Camejo G et al. (2003) Contribution of circulating triglyceride to fatty acid loading in individual tissues in vivo. Diabetes 52 [Suppl 1]:A9 (abstract)

34. Schoonjans K, Peinado-Onsurbe J, Lefebvre AM et al. (1996) PPARalpha and PPARgamma activators direct a distinct tissue-specific transcriptional response via a PPRE in the lipoprotein lipase gene. EMBO J 15:5336-5348

35. Lefebvre AM, Peinado-Onsurbe J, Leitersdorf I et al. (1997) Regulation of lipoprotein metabolism by thiazolidinediones occurs through a distinct but complementary mechanism relative to fibrates. Arterioscler Thromb Vasc Biol 17:1756-1764

36. Shachter NS (2001) Apolipoproteins C-I and C-III as important modulators of lipoprotein metabolism. Curr Opin Lipidol 12:297-304

37. Ye JM, Iglesias MA, Watson DG et al. (2003) PPARalpha/gamma ragaglitazar eliminates fatty liver and enhances insulin action in fat-fed rats in the absence of hepatomegaly. Am J Physiol 284:E531-E540 